\title{
The SNM Practice Guideline on Hepatobiliary Scintigraphy
}

A SNM Practice Guideline for Hepatobiliary Scintigraphy 4.0 (1), it was my privilege to collaborate with a dedicated group of professionals who worked tirelessly on this update. Those individuals include Brian W. Ciak (Bracco Diagnostics, Inc., Princeton, NJ), Dominique Delbeke (Vanderbilt University Medical Center, Nashville, TN), Andrew Hilson (Royal Free Hospital, London, England), Kelly Anne Holes-Lewis (Medical University of South Carolina, Charleston, SC), Michael G. Stabin (Vanderbilt University, Nashville, TN), and Harvey A. Ziessman (Johns Hopkins Outpatient Center, Baltimore, MD).

This version of the guideline has a number of substantive changes. The key departure from prior versions includes the position taken by this guideline with regard to technique for the sincalide (cholecystokinin)-stimulated gallbladder ejection fraction (GBEF) test. The new version strongly recommends a sincalide dose of $0.02 \mu \mathrm{g} / \mathrm{kg}$ infused over $60 \mathrm{~min}$. The normal GBEF with this methodology is at least $38 \%$. Unlike shorter infusion protocols, the recommended technique has the least variability in normal values and does not cause gastrointestinal side effects. The specificity for this technique is expected to be highest — and abnormal results fewest - in individuals who do not have gallbladder disease. Consequently, when the 60-min infusion technique gains wide acceptance in clinical practice, fewer patients without gallbladder disease are likely to be directed to cholecystectomy on the basis of a false-positive result.

Received Oct. 20, 2010; revision accepted Oct. 26, 2010.

For correspondence or reprints contact: Mark Tulchinsky, Division of Nuclear Medicine, Department of Radiology, P.O. Box 850, M.C. H066, Hershey, PA 17033.

E-mail: mtulchin@psu.edu

COPYRIGHT (C 2010 by the Society of Nuclear Medicine, Inc.

DOI: $10.2967 /$ jnumed.110.084475

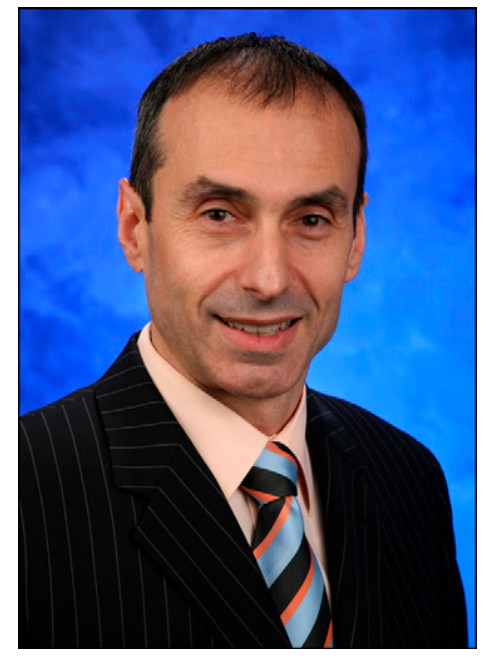

The Gastrointestinal Committee of the General Clinical Nuclear Medicine Council assembled a multidisciplinary expert panel to build consensus regarding GBEF testing. The panel met in Philadelphia on April 24, 2010, at the historic Union League building. The members included 3 nuclear medicine physicians, 3 gastroenterologists, 3 surgeons, an internist, a family medicine physician, and a nuclear medicine technologist. The panel endorsed the 60-min infusion technique and concluded that it should become the new standard in GBEF testing for patients suspected of having a functional gallbladder disorder. The panel called for uniform adoption of this technique by clinical practices. The panel's consensus manuscript, which was recently submitted for publication, should bridge the common interests of referring physicians and practicing nuclear medicine specialists for the benefit of patients.

The leadership of the General Clinical Nuclear Medicine Council intends to offer educational programs regarding the new standard in sincalide infusion technique. It would be important to understand how the GBEF test is currently done in the United States. Empiric observation suggests that the practice varies greatly, thus undermining the perceived validity of the test among referring physicians. The
Gastrointestinal Committee prepared a survey to obtain detailed information about the ways the test is done in practice, and the results of this survey will allow us to understand how much work is ahead of us. Follow-up surveys will tell us if our educational campaign is improving standardization of the test. I encourage all of you to participate in these upcoming surveys.

The indications for hepatobiliary studies were expanded from 5 general conditions in the prior version to 17 specific reasons in the current version. A section on preparation of patients referred for clinically suspected biliary atresia now includes pretreatment with ursodeoxycholic acid, as alternative to phenobarbital preparation, to increase biliary flow in patients with intrahepatic cholestasis, thus avoiding false-positive results. The dosimetry tables are updated and include available pediatric age groups and a new table for fetal doses. Although prior versions provided references in the fashion of suggested reading, this update cites relevant references for all indications, statements, and recommendations, totaling 96 references in all.

The updated guideline is comprehensive and covers many practical details, such as the information one should look for in the study request and the need to obtain this information when it is lacking. Our specialty work always benefits from detailed knowledge of the patients' clinical background, because such knowledge can lead to an individualized study protocol and can influence the study interpretation. The SNM membership is sure to find a great deal of useful and upto-date information in this guideline.

\section{Mark Tulchinsky}

Milton S. Hershey Medical Center

Pennsylvania State University

Hershey, Pennsylvania

\section{REFERENCE}

1. Tulchinsky M, Ciak BW, Delbeke D, et al. SNM practice guideline for hepatobiliary scintigraphy 4.0. J Nucl Med Technol. 2010;38:210-218. 